\title{
EXCEPTIONAL $q$-ASKEY-WILSON POLYNOMIALS AND CONTINUED FRACTIONS
}

\author{
DHARMA P. GUPTA AND DAVID R. MASSON
}

(Communicated by Kenneth R. Meyer)

\begin{abstract}
Two linearly independent solutions of the three-term recurrence relation for the $q$-Askey-Wilson polynomials are obtained for the special cases $a b c d=q^{m}, m=1,2, \ldots$. By obtaining the subdominant solution and employing Pincherle's theorem, the associated continued fractions and properties of the corresponding weight functions are derived. The cases $a b c d=q$ or $q^{2}$ are exceptional. They differ from the cases considered by Askey and Wilson [1] and are limits of a family of associated cases considered by Ismail and Rahman [5].
\end{abstract}

\section{INTRODUCTION}

Recently, Masson [8] obtained the general solution to the recurrence relation

$$
X_{n+1}-\left(z-a_{n}\right) X_{n}+b_{n}^{2} X_{n-1}=0
$$

associated with the Wilson polynomials $[13,14]$ in the special cases $a+b+c+d=$ $1,2, \ldots$. From Pincherle's theorem [6], he derived an analytical expression for the continued fraction

$$
C F(z)=z-a_{0}+K_{n=1}^{\infty}\left(\frac{-b_{n}^{2}}{z-a_{n}}\right),
$$

and also obtained the associated weight function. Here we complete the calculations indicated in [8] for the $q$-Askey-Wilson polynomials in the special cases abcd $=q^{1}, q^{2}, \ldots, 0<|q|<1$.

For associated Wilson polynomials and associated $q$-Askey-Wilson polynomials, reference may be made respectively to Masson [9], Ismail et al. [4], and Ismail and Rahman [5], where analogous results have been obtained in a more general setting depending on the properties of 2 -balanced very well poised ${ }_{7} F_{6}$ and ${ }_{8} \phi_{7}$ series.

Received by the editors December 20, 1989.

1980 Mathematics Subject Classification (1985 Revision). Primary 33A65, 30B70, 39A10, $33 \mathrm{~A} 30$.

Key words and phrases. Askey-Wilson polynomials, three-term recurrence, subdominant solution, Pincherle's theorem, continued fractions, weight functions, mass points.

Research partially supported by NSERC (Canada). 
In our derivations here we need Pincherle's theorem (see Jones and Thron [6]), from which we have the following formula for the continued fraction $C F(z)$ :

$$
\frac{1}{C F(z)}=\frac{X_{0}^{(s)}(z)}{b_{0}^{2} X_{-1}^{(s)}(z)}
$$

where $X_{n}^{(s)}$ denotes a subdominant (minimal) solution of (1).

In case of real orthogonality, $a_{n} \in \mathbb{R}, b_{n+1}^{2}>0, n=0,1,2, \ldots$, one has

$$
\frac{1}{C F(z)}=\int_{\mathbb{R}} \frac{d w(x)}{z-x}
$$

where $d w$ is a positive measure. If $x$ is in the absolutely continuous spectrum, we have (see Masson [7]) the formula

$$
w^{\prime}(x)=\frac{1}{2 \pi i} \frac{W\left(X_{-1}^{(s)}(x+i 0), X_{-1}^{(s)}(x-i 0)\right)}{b_{0}^{2}\left|X_{-1}^{(s)}(x+i 0)\right|^{2}}
$$

where $W$ is the Wronskian

$$
W\left(X_{n}, Y_{n}\right):=X_{n} Y_{n+1}-X_{n+1} Y_{n} .
$$

We shall apply (3) and (5) to the special cases of $q$-Askey-Wilson polynomials.

Note that in what follows we define indeterminate ratios in terms of limits. Thus $\left(1-q^{n+1}\right) /\left(1-q^{2 n+2}\right)$ and $\left(1-q^{n}\right) /\left(1-q^{2 n}\right)$ at $n=-1$ and 0 , respectively, are assigned the value $\frac{1}{2}$. It is this assignment which in (7), (8), and (10) below yields the exceptional cases.

\section{2. q-ASKEY-WILSON POLYNOMIALS}

The recurrence relation for $q$-Askey-Wilson polynomials [1] is given by (1) with

$$
\begin{aligned}
& b_{n}^{2}:=b_{n}^{2}(a, b, c, d ; q)=A_{n-1}(a, b, c, d ; q) B_{n}(a, b, c, d ; q), \\
& a_{n}:=a_{n}(a, b, c, d ; q)=-A_{n}(a, b, c, d ; q)-B_{n}(a, b, c, d ; q)+\frac{a}{2}+\frac{1}{2 a}, \\
& A_{n}(a, b, c, d ; q)=\frac{\left(1-a b c d q^{n-1}\right)\left(1-a b q^{n}\right)\left(1-a c q^{n}\right)\left(1-a d q^{n}\right)}{2 a\left(1-a b c d q^{2 n-1}\right)\left(1-a b c d q^{2 n}\right)}, \\
& B_{n}(a, b, c, d ; q)=\frac{a\left(1-q^{n}\right)\left(1-b c q^{n-1}\right)\left(1-b d q^{n-1}\right)\left(1-c d q^{n-1}\right)}{2\left(1-a b c d q^{2 n-2}\right)\left(1-a b c d q^{2 n-1}\right)} .
\end{aligned}
$$

The renormalized (for renormalization identities, see Masson [10]) $q$-AskeyWilson polynomial solution is given as

$$
\begin{aligned}
X_{n}^{(1)}(z ; a, b, c, d ; q)= & \frac{\left[a b c d q^{2 n-1}\right]_{\infty}}{(2 a)^{n}\left[a b c d q^{n-1}\right]_{\infty}\left[a b q^{n}, a c q^{n}, a d q^{n}\right]_{\infty}} \\
& \times{ }_{4} \phi_{3}\left(\begin{array}{c}
q^{-n}, a b c d q^{n-1}, a u, a / u ; q, q \\
a b, a c, a d
\end{array}\right),
\end{aligned}
$$


with $z=\cos \theta, u=e^{i \theta},[y]_{n}=(1-y)(1-y q) \cdots\left(1-y q^{n-1}\right),[y]_{\infty}=$ $\lim _{n \rightarrow \infty}[y]_{n}$, and $\left[y_{1}, y_{2}, \ldots, y_{k}\right]_{\infty}=\left[y_{1}\right]_{\infty}\left[y_{2}\right]_{\infty} \cdots\left[y_{k}\right]_{\infty}$.

Besides the permutation symmetry [1] with respect to the parameters $(a, b$, $c, d)$, it may be verified that

$$
\begin{aligned}
b_{-n}^{2}(q / a ; q / b, q / c, q / d ; q) & =b_{n}^{2}(a, b, c, d ; q), \\
a_{-n-1}(q / a, q / b, q / c, q / d ; q) & =a_{n}(a, b, c, d ; q) .
\end{aligned}
$$

In order to obtain a second polynomial solution of $(1)$, we apply the transformation $n \rightarrow(-n-1),(a, b, c, d) \rightarrow(q / a, q / b, q / c, q / d)$ to (1) and (8). After renormalizing, the second solution, except for a constant factor, is given by

$$
\begin{aligned}
X_{n}^{(2)}(z ; a, b, c, d ; q)= & \left(\frac{a}{2 q}\right)^{n} \frac{\left[a b c d q^{2 n-1}\right]_{\infty}}{\left[b c q^{n}, c d q^{n}, b d q^{n}\right]_{\infty}\left[q^{n+1}\right]_{\infty}} \\
& \times{ }_{4} \phi_{3}\left(\begin{array}{c}
q^{n+1}, q^{-n+2} / a b c d, q / a u, q u / a ; q, q \\
q^{2} / a b, q^{2} / a c, q^{2} / a d
\end{array}\right),
\end{aligned}
$$

$a b c d=q^{1}, q^{2}, \ldots$.

Using the subdominant solution of the next section and Pincherle's theorem, we shall prove the following in $\S 4$ :

Theorem. If $a_{n}$ and $b_{n}^{2}$ are given by (7), then the continued fraction

$$
\frac{1}{z-a_{0}+K_{n=1}^{\infty}\left(-b_{n}^{2} /\left(z-a_{n}\right)\right)}
$$

is given by (22), (23), and (24) in the respective cases abcd $=q^{2}, a b c d=q$, and abcd $=q^{m}, m=3,4, \ldots$, with $u=z+\sqrt{z^{2}-1},|u|>1$.

\section{SUbDOMINANT SOLUTION}

In order to obtain the large $n$ asymptotics of $X_{n}^{(1)}$ and $X_{n}^{(2)}$, we follow the $\mathrm{r}$-ocedure adopted by Ismail and Wilson [3]. We need the Sears transformation [11] which transforms a balanced ${ }_{4} \phi_{3}$ into another balanced ${ }_{4} \phi_{3}$ :

$$
\begin{aligned}
{ }_{4} \phi_{3}\left(\begin{array}{c}
q^{-n}, x, y, z ; q, q \\
u, v, w
\end{array}\right)= & (y z / u)^{n} \frac{[u v / y z]_{n}[u w / y z]_{n}}{[v]_{n}[w]_{n}} \\
& \times{ }_{4} \phi_{3}\left(\begin{array}{c}
q^{-n}, x, u / y, u / z ; q, q \\
u, u v / y z, u w / y z
\end{array}\right),
\end{aligned}
$$

$u v w=x y z q^{-n+1}$. When the Sears transformation is applied to $X_{n}^{(1)}$ and the relation

$$
\left[\lambda q^{-n}\right]_{k}=(-\lambda)^{k} q^{k(k-1) / 2-n k}[q / \lambda]_{n} /[q / \lambda]_{n-k}
$$

is used, then the usual argument of bounded convergence of $(n-k)$ series and Tannery's theorem (see [3, p. 47]) lead to the following asymptotic value as 
$n \rightarrow \infty:$

$$
X_{n}^{(1)} \sim\left(\frac{u}{2}\right)^{n} \frac{[a / u, b / u, c / u, d / u]_{\infty}}{[a b, a c, a d]_{\infty}\left[1 / u^{2}\right]_{\infty}}, \quad|u|>1 .
$$

For Tannery's theorem ${ }^{1}$ see [2, p. 316].

Alternatively, an interchange of $u$ and $1 / u$ gives

$$
X_{n}^{(1)} \sim\left(\frac{1}{2 u}\right)^{n} \frac{[a u, b u, c u, d u]_{\infty}}{[a b, a c, a d]_{\infty}\left[u^{2}\right]_{\infty}}, \quad|u|<1 .
$$

On the other hand, applying (11) to $X_{n}^{(2)}$ and writing $a b c d=q^{m}, m=$ $1,2, \ldots, 0<|q|<1$, we obtain the asymptotic estimate

$$
X_{n}^{(2)} \sim\left(\frac{u}{2}\right)^{n}\left(\frac{q u}{a}\right)^{m-2} \frac{[q / a u, q / b u, q / c u, q / d u]_{\infty}}{\left[q^{2} / a b, q^{2} / a c, q^{2} / a d\right]_{\infty}\left[1 / u^{2}\right]_{\infty}}, \quad|u|>1,
$$

or, alternatively,

$$
X_{n}^{(2)} \sim\left(\frac{1}{2 u}\right)^{n}\left(\frac{q}{a u}\right)^{m-2} \frac{[q u / a, q u / b, q u / c, q u / d]_{\infty}}{\left[q^{2} / a b, q^{2} / a c, q^{2} / a d\right]_{\infty}\left[u^{2}\right]_{\infty}}, \quad|u|<1 .
$$

From (13) and (15), the subdominant solution of (1) for $a b c d=q^{m}, m=$ $1,2, \ldots$, and $|u|>1$ may be written as

$$
X_{n}^{(s)}=\alpha X_{n}^{(1)}-\beta X_{n}^{(2)},
$$

where

$$
\begin{aligned}
& \alpha=\left(\frac{q u}{a}\right)^{m-2} \frac{[q / a u, q / b u, q / c u, q / d u]_{\infty}}{\left[q^{2} / a b, q^{2} / a c, q^{2} / a d\right]_{\infty}} \\
& \beta=\frac{[a / u, b / u, c / u, d / u]_{\infty}}{[a b, a c, a d]_{\infty}},
\end{aligned}
$$

with large- $n$ behavior given by

$$
\begin{aligned}
X_{n}^{(s)} \sim & \frac{(q / a)^{m-2}}{[a b, a c, a d]_{\infty}\left[q^{2} / a b, q^{2} / a c, q^{2} / a d\right]_{\infty}(2 u)^{n}\left[u^{2}\right]_{\infty}} \\
& \times\left(u^{m-2}[q / a u, q / b u, q / c u, q / d u]_{\infty}[a u, b u, c u, d u]_{\infty}\right. \\
& \left.\quad-u^{2-m}[q u / a, q u / b, q u / c, q u / d]_{\infty}[a / u, b / u, c / u, d / u]_{\infty}\right)
\end{aligned}
$$

for $|u|>1$.

\section{Particular cases}

Exceptional case I. $a b c d=q^{2}$. Computing the value of

$$
\frac{X_{n}^{(s)}(z)}{b_{n}^{2} X_{n-1}^{(s)}(z)}
$$

\footnotetext{
${ }^{1}$ Let $p(n) \uparrow \infty$ and $\lim _{n \rightarrow \infty} u_{r}(n)=v_{r}$. Then $\lim \sum_{r=0}^{p(n)} u_{r}(n)=\sum_{0}^{\infty} v_{r}$ if $\left|u_{r}(n)\right| \leq M_{r}$, $\forall n$ and $\sum^{\infty} M_{r}<\infty$.
} 
from (8), (10), and (17), and then taking $n=0$, we find that

$$
\begin{aligned}
\frac{X_{0}^{(s)}(z)}{b_{0}^{2} X_{-1}^{(s)}(z)}= & \frac{4(1-q)}{a\left(1-b c q^{-1}\right)\left(1-b d q^{-1}\right)\left(1-c d q^{-1}\right)} \\
& \times \frac{\Pi_{1}(u)-\Pi_{2}(u)}{\Pi_{1}(u)+\Pi_{2}(u)},
\end{aligned}
$$

where

$$
\begin{aligned}
& \Pi_{1}(u)=[q / a u, q / b u, q / c u, q / d u]_{\infty} \\
& \Pi_{2}(u)=[a / u, b / u, c / u, d / u]_{\infty} .
\end{aligned}
$$

Consequently, from Pincherle's theorem (3) for $|u|>1$, the following continued fraction representation follows:

$$
\begin{gathered}
\frac{4(1-q)\left[\Pi_{1}(u)-\Pi_{2}(u)\right]\left[\Pi_{1}(u)+\Pi_{2}(u)\right]^{-1}}{a\left(1-b c q^{-1}\right)\left(1-b d q^{-1}\right)\left(1-c d q^{-1}\right)} \\
=\frac{1}{z-a_{0}+K_{n=1}^{\infty}\left(-b_{n}^{2} /\left(z-a_{n}\right)\right)},
\end{gathered}
$$

with $u=z+\sqrt{z^{2}-1}$ and the branch chosen so that $|u|>1$.

Exceptional case II. $a b c d=q$. In this case, we obtain for $|u|>1$

$$
\begin{aligned}
\frac{X_{0}^{(s)}(z)}{b_{0}^{2} X_{-1}^{(s)}(z)}=4 a[ & \frac{(1-a b)(1-a c)(1-a d)}{(1-q)} \\
& \left.-(1-a u)\left(1-\frac{a}{u}\right)+a u \frac{\Pi_{2}(u)}{\Pi_{1}(u)}\right]^{-1},
\end{aligned}
$$

which gives the associated continued fraction where the left side of (22) is replaced by (23).

Note that the exceptional cases discussed above differ from the cases considered by Askey and Wilson [1]. They are $\alpha \rightarrow 0$ limits of the associated family $\left\{p_{n}^{\alpha}(x)\right\}$ examined by Ismail and Rahman [5]. The corresponding Askey-Wilson cases are $\alpha \rightarrow 0$ limits of the second associated family $\left\{q_{n}^{\alpha}(x)\right\}$ of Ismail and Rahman [5]. 
Askey-Wilson cases. abcd $=q^{m}, m=3,4, \ldots$ In these cases we have $B_{0}=0$ (and hence $b_{0}^{2}=0$ ). After some simplification, we obtain for $|u|>1$

$$
\begin{aligned}
\frac{1}{C F(z)}= & \frac{X_{0}^{(s)}(z)}{-X_{1}^{(s)}(z)+\left(z-a_{0}\right) X_{0}^{(s)}(z)} \\
= & -2 u\left(\frac{u q}{a}\right)^{m-3} \frac{[q]_{\infty}}{[a b c d]_{\infty}} \frac{\Pi_{1}(u)}{\Pi_{2}(u)} \\
& \times \frac{[b c, b d, c d]_{\infty}}{\left(1-a b q^{-1}\right)\left(1-a c q^{-1}\right)\left(1-a d q^{-1}\right)\left[q^{2} / a b, q^{2} / a c, q^{2} / a d\right]_{\infty}} \\
& +\left(\frac{2 a}{q}\right) \frac{\left(1-q^{m-1}\right)}{\left(1-a b q^{-1}\right)\left(1-a c q^{-1}\right)\left(1-a d q^{-1}\right)} \\
& \times{ }_{4} \phi_{3}\left(\begin{array}{c}
q^{2} / a b c d, q, q / a u, q u / a ; q, q \\
q^{2} / a b, q^{2} / a c, q^{2} / a d
\end{array}\right) .
\end{aligned}
$$

\section{WEIGHT FUNCTION}

Once we have the continued fractions (22), (23), (24), we may use (5) to obtain the absolutely continuous part of the weight function in the respective cases. We obtain the following expressions for the weight function: When $a b c d=q^{2}$,

$$
\begin{aligned}
w^{\prime}(x)= & \frac{1}{\pi i} \frac{4(1-q)}{a\left(1-b c q^{-1}\right)\left(1-b d q^{-1}\right)\left(1-c d q^{-1}\right)} \\
& \times \frac{\Pi_{1}(1 / u) \Pi_{2}(u)-\Pi_{1}(u) \Pi_{2}(1 / u)}{\left|\Pi_{1}(u)+\Pi_{2}(u)\right|^{2}} ;
\end{aligned}
$$

when $a b c d=q$,

$$
\begin{aligned}
w^{\prime}(x)= & \frac{2 a^{2}}{\pi i}\left[\frac{u \Pi_{2}(u)}{\Pi_{1}(u)}-\frac{\Pi_{2}(1 / u)}{u \Pi_{1}(1 / u)}\right] \\
& \times\left|\frac{(1-a b)(1-a c)(1-a d)}{(1-q)}-(1-a u)\left(1-\frac{a}{u}\right)+a u \frac{\Pi_{2}(u)}{\Pi_{1}(u)}\right|^{-2} ;
\end{aligned}
$$

and when $a b c d=q^{m}, m=3,4, \ldots$,

$$
\begin{aligned}
w^{\prime}(x)= & \frac{1}{\pi i}\left(\frac{q}{a}\right)^{m-3} \frac{[q]_{\infty}}{[a b c d]_{\infty}} \\
& \times \frac{[b c, b d, c d]_{\infty}}{\left(1-a b q^{-1}\right)\left(1-a c q^{-1}\right)\left(1-a d q^{-1}\right)\left[q^{2} / a b, q^{2} / a c, q^{2} / a d\right]_{\infty}} \\
& \times\left[u^{m-2} \frac{\Pi_{1}(u)}{\Pi_{2}(u)}-u^{2-m} \frac{\Pi_{1}(1 / u)}{\Pi_{2}(1 / u)}\right]
\end{aligned}
$$

$u=x+i \sqrt{1-x^{2}}, x \in(-1,1)$. 
Note that, in order to accommodate the case of complex measures, we have used in (25) and (26) the convention $|f(u)|^{2}=f(u) f(1 / u)$ so that complex conjugation is taken only with respect to the variable $u=x+i \sqrt{1-x^{2}}$.

The cases $a b c d=q^{m}, m=3,4, \ldots$, are special $q$-Askey-Wilson cases. To see that (27) coincides with the weight function obtained by Askey and Wilson [1], we derive an identity using an alternative calculation of the $W$ in (6).

By iterative use of (1), we can derive

$$
b_{0}^{2} W\left(X_{-1}^{(s)}(x+i 0), X_{-1}^{(s)}(x-i 0)\right)=\lim _{n \rightarrow \infty} \frac{W\left(X_{n}^{(s)}(x+i 0), X_{n}^{(s)}(x-i 0)\right)}{b_{1}^{2} b_{2}^{2} \cdots b_{n}^{2}}
$$

From (28), we obtain the following modification of (5):

$$
w^{\prime}(x)=\frac{1}{2 \pi i} \lim _{n \rightarrow \infty} \frac{W\left(X_{n}^{(s)}(x+i 0), X_{n}^{(s)}(x-i 0)\right)}{b_{1}^{2} b_{2}^{2} \cdots b_{n}^{2} \cdot b_{0}^{4}\left|X_{-1}^{(s)}(x+i 0)\right|^{2}} .
$$

Using (29) and the asymptotic value of $X_{n}^{(s)}$ given by (19), we obtain, for abcd $=q^{m}, m=3,4, \ldots$,

$$
\begin{aligned}
w^{\prime}(x)= & \frac{1}{\pi i}\left(\frac{q}{a}\right)^{2 m-6}\left(\frac{1}{u}-u\right) \\
& \times \frac{[b c, b d, c d]_{\infty}[q]_{m-1}\left[\left(1-a b q^{-1}\right)\left(1-a c q^{-1}\right)\left(1-a d q^{-1}\right)\right]^{-2}}{\left[u^{2}\right]_{\infty}\left[1 / u^{2}\right]_{\infty}\left[\frac{q^{2}}{a b}, \frac{q^{2}}{a c}, \frac{q^{2}}{a d}\right]_{\infty}^{2} \Pi_{2}(u) \Pi_{2}(1 / u)[a b, a c, a d]_{\infty}} \\
& \times\left[u^{m-2} \Pi_{1}(u) \Pi_{2}(1 / u)-u^{2-m} \Pi_{1}(1 / u) \Pi_{2}(u)\right]^{2} .
\end{aligned}
$$

Equating values of $w^{\prime}(x)$ given by (27) and (30), we obtain the nontrivial identity

$$
\begin{aligned}
\left(\frac{q}{a}\right)^{m-3} & \left(\frac{1}{u}-u\right)\left[u^{m-2} \Pi_{1}(u) \Pi_{2}(1 / u)-u^{2-m} \Pi_{1}(1 / u) \Pi_{2}(u)\right] \\
= & {\left[a b q^{-1}, a c q^{-1}, a d q^{-1}\right]_{\infty}\left[\frac{q^{2}}{a b}, \frac{q^{2}}{a c}, \frac{q^{2}}{a d}\right]_{\infty}\left[u^{2}\right]_{\infty}\left[\frac{1}{u^{2}}\right]_{\infty} }
\end{aligned}
$$

for $m=3,4, \ldots,|u|=1$.

From (27) and the above identity we recover the Askey-Wilson form of the weight function (see [1, pp. 11-12]), viz.,

$$
w^{\prime}(x)=\frac{1}{2 \pi \sqrt{1-x^{2}}} \frac{[a b, a c, a d]_{\infty}[b c, b d, c d]_{\infty}[q]_{\infty}\left[u^{2}\right]_{\infty}\left[1 / u^{2}\right]_{\infty}}{[a u, b u, c u, d u]_{\infty}[a / u, b / u, c / u, d / u]_{\infty}[a b c d]_{\infty}} .
$$

Here we have given an almost algebraic proof of (32). The derivation is independent of the usual orthogonality conditions $a_{n} \in \mathbb{R}, b_{n+1}^{2}>0$ and for more general parameter values gives us the absolutely continuous part of the complex measure whose Stieltjes transform gives the continued fraction (24). This complex measure is purely absolutely continuous if $|a|,|b|,|c|,|d|<1$, since the discrete part is given by the zeros of $\Pi_{2}(u)$ in (24). 
Repeating the calculation for the exceptional cases $a b c d=q$ or $q^{2}$ establishes the identity (31) for the additional cases $m=1,2$. Note that identity (31) is a special case of the $q$-analogue of Dougall's theorem [9].

For comparison, we record the corresponding alternative form for the weight function in the exceptional cases. Using (26), (27), and (31), we obtain the following results:

Case abcd $=q^{2}$.

$$
w^{\prime}(x)=\frac{2(1-q)}{\pi \sqrt{1-x^{2}}} \frac{[a b, a c, a d, b c, b d, c d]_{\infty}\left[u^{2}\right]_{\infty}\left[u^{-2}\right]_{\infty}}{\left|\Pi_{1}(u)+\Pi_{2}(u)\right|^{2}} .
$$

Case $a b c d=q$.

$$
\begin{aligned}
& w^{\prime}(x)=\frac{a^{2}}{\pi \sqrt{1-x^{2}}} \\
& \quad \times \frac{[a b, a c, a d, b c, b d, c d]_{\infty}\left[u^{2}\right]_{\infty}\left[u^{-2}\right]_{\infty}}{\left|\Pi_{1}(u)[(1-a b)(1-a c)(1-a d) /(1-q)-(1-a u)(1-a / u)]+a u \Pi_{2}(u)\right|^{2}} .
\end{aligned}
$$

In the next section we discuss the discrete spectrum in the case when the measure is positive.

\section{MASS POINTS}

We consider here some properties of the discrete spectrum in the case of real orthogonality $\left(a_{n} \in \mathbb{R}, b_{n+1}^{2}>0, n \geq 0\right)$.

In the normal Askey-Wilson cases, (24) yields an explicit discrete spectrum from the zeros of $\Pi_{2}(u)$. Thus there are no mass points if $-1<a, b, c, d<1$. Mass points exist in $(1, \infty)$ for $0<|q|<1$ if one of the parameters is greater than unity, and in $(-\infty,-1)$ if one of the parameters is less than -1 .

For the exceptional cases, the discrete spectrum is no longer explicit.

Exceptional case I. From (22), when $a b c d=q^{2}$, we have to examine the function

$$
\frac{\Pi_{1}(u)-\Pi_{2}(u)}{\Pi_{1}(u)+\Pi_{2}(u)} .
$$

It is clear that no mass points exist for $0<|q|<1,|q|<|a|,|b|,|c|$, $|d|<1$, because of the positivity of $\Pi_{1}(u)+\Pi_{2}(u)$.

On the other hand, we will obtain the existence of mass points using the monotonicity of (4) for a positive measure, i.e.,

$$
\frac{d}{d z} \int \frac{d w(x)}{z-x}=-\int \frac{d w(x)}{(z-x)^{2}}<0, \quad z \in \mathbb{R}, \quad z \notin \operatorname{supp} d w .
$$

With an absolutely continuous spectrum in $[-1,1]$ this monotonicity implies that a zero of $(22)$ as a function of $u$ (or $z$ ) outside the interval $[-1,1]$ is accompanied by a pole. 
We now observe that mass points exist:

(i) if both $\Pi_{1}(u)$ and $\Pi_{2}(u)$ have a zero either for $u>1$ or for $u<-1$, e.g., when $a>1$ and $0<b<|q|$, or when $a<-1,-|q|<b<0$;

(ii) if either $\Pi_{1}(u)$ or $\Pi_{2}(u)$ has more than one zero in $\mathbb{R} \backslash[-1,1]$, e.g., for $a>1, b<-1$, or $a>1, b>1$, or $a>1 / q, q>0$, or $0<a<q^{2}$, $q>0$, or $a>-1 / q^{2}, q<0$, etc.

To obtain (i), suppose $\Pi_{1}(u)=0$ for some value of $u$, say $u_{1}>1$. Then for $u=u_{1}>1,1 / C F(z)=A$,

$$
A:=\frac{-4(1-q)}{a\left(1-b c q^{-1}\right)\left(1-b d q^{-1}\right)\left(1-c d q^{-1}\right)} .
$$

If in addition $\Pi_{2}(u)=0$ for some value of $u$, say $u_{2}>1$, then for $u=u_{2}>1$, $1 / C F(z)=-A$. Consequently, there exists a zero of $1 / C F(z)$ between $u_{1}$ and $u_{2}$, say at $u=u^{*}>1$. Hence there exists a pole of $1 / C F(z)$ at some $u^{* *}>u^{*}$.

To obtain (ii), suppose $\Pi_{1}(u)$ has two zeros, $u^{\prime}, u^{\prime \prime}$ in $\mathbb{R} \backslash[-1,1]$. Then $1 / C F(z)=A$ at both $u^{\prime}$ and $u^{\prime \prime}$. The monotonicity implies a zero between $u^{\prime}$ and $u^{\prime \prime}$ and consequently a pole.

Note that, in contrast with the Askey-Wilson cases, we can now have a mass point with all parameters in the interval $(-1,1)$. This can be seen by first considering the case $a=q^{2}, b=c=d=1$, which yields $\Pi_{1}(u)=$ $[1 / q u, q / u, q / u, q / u]_{\infty}$ and $\Pi_{2}(u)=\left[q^{2} / u, 1 / u, 1 / u, 1 / u\right]_{\infty}$. Thus

$$
\begin{aligned}
\Pi_{1}(u)+\Pi_{2}(u)= & {\left[(1-q / u)(1-1 / q u)+(1-1 / u)^{2}\right] } \\
& \times\left[1 / u, q^{2} / u, q / u, q / u\right]_{\infty},
\end{aligned}
$$

which has a zero at $z=\frac{1}{2}\left(u+u^{-1}\right)=(1+q)^{2} / 4 q>1$, if $0<q<1$. By continuity $\Pi_{1}(u)+\Pi_{2}(u)$ will have a zero for some $z>1(u>1)$ if $a=q^{2-\varepsilon}, b=c=d=q^{\varepsilon / 3}$, with $\varepsilon$ small and positive. Alternatively, since it can be shown that for this case $a_{0}>1$, so that $P_{1}(z)=z-a_{0}$ has a zero for $z=a_{0}>1$, we can use the result in Szegö [12, Theorem 3.3.1]. By a similar argument, one gets a zero in the denominator of (22) for some $z<-1$ $(u<-1)$ if $a=-|q|^{2-\varepsilon}, b=c=d=-|q|^{\varepsilon / 3}$, and $-1<q<0$.

Also, by perturbing the case $a=1, b=c=d=q^{2 / 3}$ if $0<q<1$ or the case $a=-1, b=c=d=|q|^{2 / 3}$ if $-1<q<0$, we can see that it is no longer sufficient to have a parameter outside the interval $(-1,1)$ in order to obtain a mass point.

The above properties will be true also for the family $\left\{p_{n}^{\alpha}(x)\right\}$ of associated Askey-Wilson polynomials discussed by Ismail and Rahman [5] if $\alpha$ is sufficiently small and abcd is sufficiently close to $q^{2}$.

Exceptional case II. Finally we discuss the mass points in the exceptional case when $a b c d=q$. For expression (23), we prove the nonexistence of mass points for the normal range of the parameters $-1<a, b, c, d<1$. 
First consider $q<a, b, c, d<1,0<q<1, u>1$. The denominator in (23) will always be positive if $(1-a d)(1-a c)(1-a b) /(1-q)-\left(1-a u-a / u+a^{2}\right)>$ 0 . Since $1-a u-a / u+a^{2}=1-2 a z+a^{2}<(1-a)^{2}$, the condition may be written as

$$
2 a\left[\frac{(1-a d)(1-a c)((1-a b)}{2 a(1-q)}-\frac{1}{2 a}-\frac{a}{2}\right]+2 a>0,
$$

or $-2 a a_{0}+2 a>0$, where $a_{0}$ is given by (7). Thus positivity will follow for $a_{0}<1$. But, for $a b c d=q$,

$$
a_{0}=(a+b+c+d-q / a-q / b-q / c-q / d) / 2(1-q) .
$$

Examining the maximum value of $a_{0}$ for $q \leq a, b, c, d \leq 1$, with the constraint $a b c d=q$, we find that the maximum $a_{0}=1$ occurs when two of the parameters are equal to 1 . Thus there are no mass points for $q<a, b, c, d<1$, $0<q<1$. However, because of the constraint $a b c d=q$, if one has $0<a \leq q$ and $b, c, d>0$, this implies that some parameter, say $d$, satisfies $d \geq 1$. Thus, it is sufficient to require $0<a, b, c, d<1$.

If one parameter, say $a$, is negative and $u>1$, one can similarly see that the denominator in (23) will always be negative if $a_{0}>-1$, and one can examine the minimum of $a_{0}$. By similarly considering the cases $u<-1$ and $-1<q<0$, we show that there are no mass points if $-1<a, b, c, d<1$.

Alternatively, one can realize that $a_{0}=1 / 2 a+a / 2-A_{0}-B_{0}$ in this exceptional case is the same as in the regular Askey-Wilson case. (Here we have $A_{0}=B_{0}$ while the corresponding Askey-Wilson case replaces $A_{0}$ by $2 A_{0}$ and $B_{0}$ by 0. .) Thus, the absence of mass points in the Askey-Wilson case when $-1<a, b, c, d<1$ implies that $-1<a_{0}<1$, which in turn implies the absence of mass points for this exceptional case because of the positive or negative definiteness of the denominator in (23).

For the existence of a mass point in the exceptional case $a b c d=q$, it suffices to have some real parameter, say $a$, satisfying $|a|<|q|$, since this implies that $\Pi_{1}(u)=0$ for some real $u,|u|>1$, which yields a zero in (23). This in turn implies a pole in (23) by the monotonicity property mentioned in the case $a b c d=q^{2}$. However, we note that, in contrast to the Askey-Wilson cases, it is no longer sufficient to have some parameter, say $a$, satisfying $a>1$ or $a<-1$ in order to have mass points. To see this one can perturb the case $|a|=1$, $|b|=|c|=|d|=|q|^{1 / 3}$, which has no mass points.

\section{REFERENCES}

1. R. Askey and J. Wilson, Some basic hypergeometric orthogonal polynomials that generalize Jacobi polynomials, Mem. Amer. Math. Soc., No. 319, Amer. Math. Soc., Providence, RI, 1985 , pp. $1-55$.

2. T. J. I'A. Bromwich, An introduction to the theory of infinite series, 2nd ed., Macmillan, New York, 1955.

3. M. E. H. Ismail and J. A. Wilson, Asymptotic and generating relations for the $q$-Jacobi and ${ }_{4} \phi_{3}$ polynomials, J. Approx. Theory 36 (1982), 43-54. 
4. M. E. H. Ismail, J. Letessier, G. Valent, and J. Wimp, Two families of associated Wilson polynomials, Canad. J. Math. 42 (1990), 659-695.

5. M. E. H. Ismail and M. Rahman, The associated Askey-Wilson polynomials, Trans. Amer. Math. Soc. (to appear).

6. W. B. Jones and W. J. Thron, Continued fractions: Analytic theory and applications, Addison-Wesley, Reading, MA, 1980.

7. D. R. Masson, Difference equations, continued fractions, Jacobi matrices and orthogonal polynomials, in Non-linear numerical methods and rational approximation (A. Cuyt, ed.), Reidel, Dordrecht, 1988, pp. 239-257.

8. Wilson polynomials and some continued fractions of Ramanujan, Rocky Mountain J. Math. (to appear).

9. __ Associated Wilson polynomials, Constructive Approximation (to appear).

10. _ Difference equations revisited, Canad. Math. Soc. Conf. Proc. (J. S. Feldman and L. M. Rosen, eds.), vol. 9, Amer. Math. Soc., Providence, RI, 1988, pp. 73-82.

11. D. Sears, On the transformation theory of basic hypergeometric functions, Proc. London Math. Soc. (2) 53 (1951), 158-180.

12. G. Szegö, Orthogonal polynomials, Amer. Math. Soc., Providence, RI, 1975.

13. J. A. Wilson, Three-term contiguous relations and some new orthogonal polynomials, Padé and Rational Approximation (Proc. Internat. Sympos. Univ. South Florida, 1976), Academic Press, New York, 1977, pp. 227-232.

14. J. Wilson, Hypergeometric series, recurrence relations and some orthogonal functions, Ph.D. diss., University of Wisconsin, Madison, 1978.

Department of Mathematics, University of Toronto, Toronto M5S 1A1, Canada 